\title{
Antibiotic consumption and ventilator-associated pneumonia rates, some parallelism but some discrepancies
}

\author{
David Nora $^{1,2}$, Pedro Póvoa ${ }^{1,2}$ \\ ${ }^{1}$ Polyvalent Intensive Care Unit, Hospital de São Francisco Xavier, Centro Hospitalar de Lisboa Ocidental, Lisbon, Portugal; ${ }^{2}$ NOVA Medical \\ School, New University of Lisbon, Lisbon, Portugal \\ Contributions: (I) Conception and design: All authors; (II) Administrative support: None; (III) Provision of study materials or patients: All authors; (IV) \\ Collection and assembly of data: D Nora; (V) Data analysis and interpretation: All authors; (VI) Manuscript writing: All authors; (VII) Final approval \\ of manuscript: All authors. \\ Correspondence to: David Nora. Unidade de Cuidados Intensivos Polivalente-Piso 1, Hospital de São Francisco Xavier, Estrada do Forte do Alto do \\ Duque, 1449-005 Lisboa, Portugal. Email: davidjgarcianora@gmail.com.
}

\begin{abstract}
Ventilator-associated pneumonia (VAP) is a common infection in intensive care units (ICUs) but its clinical definition is neither sensitive nor specific and lacks accuracy and objectivity. New defining criteria were proposed in 2013 by the National Healthcare Safety Network (NHSN) in order to more accurately conduct surveillance and track prevention progress. Although there is a consistent trend towards a decrease in VAP incidence during the last decade, significant differences in VAP rates have been reported and are persistently lower in NHSN and other American reports ( 0.0 to 4.4 VAP per 1,000 ventilator-days in 2012) compared to the European Centre for Disease Prevention and Control (ECDC) data (10 VAP per 1,000 ventilator-days in 2014). In the United States, VAP has been proposed as an indicator of quality of care in public reporting, and the threat of financial penalties for this diagnosis has put pressure on hospitals to minimize VAP rates that may lead to artificial lower values, independently of patient care. Although prevention bundles may contribute for encouraging reductions in VAP incidence, both pathophysiologic and epidemiologic factors preclude a zero-VAP rate. It would be expected from the trend of reduction of VAP incidence that the consumption of antibiotics would also decrease in particular in those hospitals with lowest VAP rates. However, ICU reports show a steadily use of antibiotics for nosocomial pneumonia in $15 \%$ of patients and both ECDC and NHSN data on antibiotic consumption showed no significant trend. Knowledge of bacterial epidemiology and resistance profiles for each ICU has great relevance in order to understand trends of antibiotic use. The new NHSN criteria provide a more objective and quantitative data based VAP definition, including an antibiotic administration criterion, allowing, in theory, a more comprehensive assessment and a reportable benchmark of the observed VAP and antibiotic consumption variability.
\end{abstract}

Keywords: Antibiotic; intensive care unit (ICU); ventilator-associated pneumonia (VAP)

Submitted Sep 06, 2017. Accepted for publication Sep 08, 2017.

doi: 10.21037/atm.2017.09.16

View this article at: http://dx.doi.org/10.21037/atm.2017.09.16

\section{Introduction}

Ventilator-associated pneumonia (VAP) is one of the most common health care-associated infections (HAI) and accounts for $25 \%$ to $42 \%$ of all infections that occur in intensive care units (ICUs) (1-3). It can hit $10 \%$ to $25 \%$ of all ICU patients $(4,5)$, being associated with longer duration of mechanical ventilation, longer ICU and hospital stays, increased healthcare costs and increased morbidity and mortality $(6,7)$. VAP mortality rates range from $20 \%$ to $70 \%$ with healthcare costs of $\$ 20,000$ to $\$ 40,000$ per patient $(4,8)$, making it a primary focus for risk-reduction strategies (9). In recent years preventive measures have been implemented and disseminated to reduce both its incidence 
Table 1 VAP definition criteria evolution $(10,17)$

\begin{tabular}{|c|c|c|}
\hline Variables & Criteria before 2013 & Criteria after 2013 \\
\hline \multicolumn{3}{|c|}{$\begin{array}{l}{ }^{\dagger} \text {, must follow a period } \geq 2 \text { days of stable or decreasing daily minimum PEEP or daily minimum } \mathrm{FiO}_{2} \text { and persist for } \geq 2 \text { days; }{ }^{\ddagger}, \text { the presence } \\
\text { of at least one of these respiratory parameters constitutes a VAC; }{ }^{\S} \text {, the presence of VAC plus one of these infection or inflammation } \\
\text { parameters constitutes a IVAC; }{ }^{n} \text {, the presence of IVAC plus one of this parameter constitutes a possible pneumonia; }{ }^{\phi}, \text { the presence of } \\
\text { IVAC with both a positive Gram's staining and a positive quantitative culture constitutes a probable pneumonia. WBC, white blood cell; } \\
\text { ETT/MV, endotracheal intubation/mechanical ventilation; CFU, colony-forming unit; } \mathrm{FiO}_{2} \text {, inspired fraction of oxygen; PEEP, positive end } \\
\text { expiratory pressure. }\end{array}$} \\
\hline
\end{tabular}

and its consequences (10) and VAP has become a mean for tracking quality of care for ventilated patients (11).

\section{VAP definition}

Despite VAP epidemiological impact, it has no valid and reliable definition and even the most widely used VAP criteria are neither sensitive nor specific (6) and lack accuracy and objectivity $(7,12,13)$, with documented poor correlation between the clinical diagnosis of VAP and histologic examination (14). Clinical scores have been applied but none was consistent in accurately identifying VAP (15). From 2002 onwards, the National Healthcare Safety Network (NHSN) criteria for VAP diagnosis include a combination of X-ray, signs or symptoms and laboratory data (16) (Table 1) in patients who were tracheally intubated and under mechanically ventilation at some time (with no minimal required amount of time) during the $48 \mathrm{~h}$ preceding the onset of pneumonia. Clinically, VAP can be subdivided into early and late onset: early onset VAP occurs within the first 4 days and late onset VAP after 4 days of mechanical ventilation $(15,18)$. Both the subjectivity and variability inherent to chest $\mathrm{X}$-ray technique and interpretation and to signs or symptoms recognition and proper documentation in medical records make these criteria unreliable to accurately identify VAP (6).

This is far from ideal to conduct surveillance and track prevention progress in an era of public reporting of HAI rates, benchmarking, inter-facility comparisons, and payfor-reporting and pay-for-performance programs. As an example, simply changing the microbiological method of diagnosis within the context of normal clinical practice influences VAP rates and antibiotic use. Morris et al. (19) compared the diagnosis of VAP in 53 patients with clinical suspicion using endotracheal aspirate (ETA) and bronchoalveolar lavage (BAL) and found that ETA overdiagnosed VAP compared with BAL (89\% versus $21 \%$ of clinically suspected cases, $\mathrm{P}<0.0001)$. Using infection surveillance data of 643 patients, 110 of which with clinically suspected VAP, Morris et al. (19) modelled the potential effect on VAP incidence and antibiotic use of using exclusively ETA or BAL and found that changing from exclusive ETA to BAL diagnosis would decrease reported VAP incidence by $76 \%$ (95\% CI, $67 \%$ to $87 \%$ ) and antibiotic use by $30 \%$ (95\% CI, 20\% to $42 \%$ ).

In 2013, the Centers for Disease Control and Prevention (CDC) brought together a multidisciplinary team of renowned experts to address the limitations of the NHSN definition and propose a new set of metrics (6). Therefore, a tiered definition algorithm was developed with three main targets: first, to extend surveillance to generic complications of mechanical ventilation (both infectious and non-infectious); second, to make surveillance as objective as possible; third, to enable an automatic enrollment of clinical records. It emphasizes the importance of preventing all complications of mechanical ventilation rather than pneumonia alone (20), introducing the concept of ventilator-associated event (VAE). VAE consists of ventilator-associated conditions (VAC), infection-related ventilator-associated complications (IVAC) (a subset of 
VAC with infectious signs), possible and probable VAP (IVAC with microbiological evidence of pneumonia) that are characterized based solely on quantitative (and so objective) criteria (Table 1). Such tiers and definitions are only suitable for potential use in public reporting, benchmarking or internal quality improvements initiatives and are not designed for use in the management of patients. As a practical example, they completely disregard radiographic criteria, not its role in daily practice, but its lack of objectivity and accuracy to constitute a useful tool in surveillance reports (17).

\section{VAP rates surveillance}

Significant differences in VAP rates have been reported in the literature (21).

The International Nosocomial Infection Control Consortium (INICC) conducted a cohort prospective multicentre surveillance study of device associated HAI in 703 ICUs in 50 countries from Latin America, Europe, Eastern Mediterranean, Southeast Asia, and Western Pacific World Health Organization regions (United States not included) from 2010 to 2015 (22). They found a pooled mean VAP rate (number of VAP over 1,000 ventilation-days) of 12.2 (95\% CI, 12.0-12.4) with lower incidence in surgical cardiothoracic ICUs (6.2, 95\% CI, 5.5-7.0) and higher incidence in neurologic ICU (19.2, 95\% CI, 15.6-23.5). The pooled mean of ventilator used ratio (ventilator-days per 100 patient-days) was 37 (95\% CI, 37-37), being lower in medical cardiac ICU (20, 95\% CI, 20-20) and higher in trauma ICU (66, 95\% CI, 65-66). A significant trend toward the reduction of VAP rates in INICC ICUs was observed since 2002, when surveillance reports presented a VAP rate of 24.1 (95\% CI, 22.8-25.5), virtually twice the observed VAP rate in the 2010-2015 period.

Martin-Loeches et al. (5) prospectively analyzed almost 3000 ICU patients from 114 ICUs in Europe and South America during 2013 and 2014 and found an incidence of VAP of 8.8 per 1,000 ventilation-days that was statistically similar to the incidence of ventilator-associated tracheobronchitis (VAT) (10.2 per 1,000 mechanically ventilated days, $\mathrm{P}=0.48)$. Trauma patients had a higher proportion of VAP and VAT (18\% and $19 \%)$ when compared to both medical (9\% and 12\%) and surgical ( $9 \%$ and $10 \%)$ patients, which had already been described in previous studies (23).

When comparing these results with the United States (US) surveillance data reported by the NHSN the differences are quite significant, with much higher VAP rates in the INICC report, in some case more than 20 times $(22,24)$. The most recent NHSN report (from 2012) found pooled VAP rates of 0.0 to 4.4 with pooled mean ventilator used ratio of 3 to 47 ventilator-days per 100 patient-days (24). Making a direct comparison by ICU-type we found in surgical cardiothoracic ICUs an INICC VAP rate of $6.2(95 \%$ CI, 5.5-7.0) versus a NHSN VAP rate of 1.7 (95\% CI, 1.5-1.9), in neurologic ICU an INICC VAP rate of 19.2 (95\% CI, 15.6-23.5) versus a NHSN VAP rate of 3.0 (95\% CI, 2.3-3.8) and in respiratory ICU an INICC VAP rate of 13.8 (95\% CI, 11.9-15.8) versus a NHSN VAP rate of 0.7 (95\% CI, 0.2-1.7) (22). Some differences may clearly reflect socioeconomic contrasts leading to differences in daily clinical practices, namely a possible irregular adherence to and compliance with the guidelines, low nurse-to-patient staffing ratios, lack of medical supplies or an insufficient number of experienced nurses or trained health care workers (22).

Deeper examining the NHSN reported data, there is a $13 \%$ to $19 \%$ per year reduction in VAP rates from 2002 to 2008 (25) and more than $50 \%$ of non-teaching medical ICUs in the United States are reporting VAP rates of zero since 2010, with higher mean rates in surgical units (2.5 VAP per 1,000 ventilator-days) versus medical units (1.0 VAP per 1,000 ventilator-days) (20,24,26,27). Enthusiasm fades and curiosity sets in when comparing these data with European results, where socioeconomic conditions and sophisticated prevention and care programs are similar to the United States. In 2014, the European Centre for Disease Prevention and Control (ECDC) described in patient-based surveillance, a pooled rate of 10.0 VAP episodes per 1,000 intubation-days that varied between 2.8 in the United Kingdom and 15.8 in Italy and Belgium, with ventilator used ratio between 32.8 ventilator-days per 100 patient-days in Luxembourg and 68.2 ventilator-days per 100 patient-days in Portugal (28). These rates remained stable compared with 2011 and 2012 (29) and represent a $25 \%$ reduction of pooled VAP rate since 2007 (30).

These significant differences between NHSN and INICC/ ECDC data raise the question to what extent the NHSN numbers reflect real improvements in care rather than surveillance biases (17). In the United States, VAP has been proposed as an indicator of quality of care in public reporting, and its prevention is a national patient safety goal (15). The threat of financial penalties for this diagnosis has put pressure on hospitals to minimize VAP rates (18) which may prompt surveyors to apply subjective criteria more strictly, 
leading to misleading lower rates, independently of patient care. Klompas (31) described 8 initiatives that hospitals might be considering applying to make VAP surveillance more rigorous but which will artificially lower VAP rates despite not materially improving prevention and care, regardless of how well intentioned those measures might be. Such initiatives included interpretation of clinical signs and chest $\mathrm{X}$-ray as strictly as possible, require consensus between two or more infection preventionists and intensivists, require BAL cultures for diagnosis, set quantitative growth thresholds for BAL and ETA cultures, transfer out patients needing prolonged mechanical ventilation and admit uncomplicated postoperative mechanically ventilated patients (31). This makes evident the high variability and how artificial or manipulated the rates might be (32) and enables the fact that clinicians who track VAP in parallel with their hospitals' infection surveyors consistently find more cases than the surveyors do (17).

\section{VAP rates and antibiotic consumption}

Optimal antibiotic therapy is a major focus of VAP treatment (33). Both inappropriate initial antimicrobial treatment and delays in the administration of appropriate antibiotic therapy are associated with higher mortality and longer hospital stays in ICU patients $(33,34)$. Due to these observations, a number of clinical practice guidelines for antibiotic therapy for VAP have evolved over the years $(34,35)$. The optimal duration of antimicrobial treatment for VAP is unknown but there is a growing evidence that shorter antibiotic courses of 6-8 days can be effective and simultaneously minimize the consequences of antibiotic overuse in critical care, including antibiotic resistance, adverse effects and costs $(35,36)$.

It would be expected from the evidence of benefit towards shorter courses of antibiotics associated with the reduction of VAP rate that the consumption of antibiotics would also decrease in particular in those hospitals with VAP rate of "zero". Ferreira et al. (2) evaluated the clinical impact of a good practice bundle in ICU patients and found a decreased occurrence of VAP $(\mathrm{P}<0.01)$, a significant reduction in hospital costs $(\mathrm{P}<0.05)$ and lower mortality $(\mathrm{P}<0.01)$ as well as significantly less days of antibiotic use $(\mathrm{P}<0.0001)$. Rosenthal et al. (37) demonstrated the effectiveness of a multidimensional approach for prevention of VAP in adult ICUs (VAP rate evolved from 22.0 to 17.2, $\mathrm{P}=0.0004)$ and a simultaneous pooled reduction in antibiotic consumption. Primary analysis revealed a significant increase in the ratio of patients free of antibiotics comparing both periods ( $36.2 \%$ to $40.9 \%, \mathrm{P}=0.0002$ ), with a differential decrease in consumption of ciprofloxacin (6.6\% to $5.6 \%, \mathrm{P}=0.0087)$ and ceftriaxone $(17.7 \%$ to $12.1 \%, \mathrm{P}=0.0001)$. When analyzed defined daily dose (DDD) per 1,000 patient-days (DDD/1,000 PD), a similar reduction in antibiotic prescription was noticed during intervention period (958.3 to $925.8 \mathrm{DDD} / 1,000 \mathrm{PD}$ ). It was found a reduction in the administration of five groups of antibiotics-cephalosporins, quinolones, lincosamides, glycopeptides, and aminoglycosides-and a rise of carbapenems and penicillins (38).

This data however is not consistent with ICU reports that show a steadily use of antibiotics for nosocomial pneumonia in $15 \%$ of patients (17). Fihman et al. (1) retrospectively analyzed a 5 -year trend of ICU antibiotic consumption and resistance in bacteria causing VAP between 2007 and 2011 in a university-affiliated tertiary care institution in France. The frequency of early VAP episodes decreased significantly over the study period $(\mathrm{P}<0.011)$ but antibiotic consumption rose by more than one-third during this period $(1,004$ to $1,389 \mathrm{DDD} / 1,000 \mathrm{PD})$. This change though was not statistically significant $(\mathrm{P}=0.07)$ and is comparable to the median (range) antibiotic consumption of 1,254 (range, 348-4,992) DDD/1,000 PD reported by Hanberger et al. (39) in a European ICU multicenter surveillance program during 2005.

ECDC 2007 surveillance report showed that 77.4\% of patients received at least one systemic antibiotic while in the ICU. Since information on dosage is not collected in the surveillance protocol, antimicrobial consumption was expressed as the number of ICU days with at least one antimicrobial administered (two different antimicrobials on one day $=1$ day) -67.5 antibiotic days per 100 patient days-as well as the total number of antimicrobial days (two different antimicrobials on 1 day $=2$ days) per 100 patient days in the ICU-131.8 antibiotic days per 100 patient days (30). During the biennium 2011-2012 the ECDC reported a prevalence of antimicrobial use among ICU patients of $56.5 \%$ and $37.3 \%$ of all antibiotics used to treat HAI were used in ICU patients (37.3\%). Tracheal intubation was an independent risk factor for antibiotic consumption (adjusted odds ratio 2.1, 95\% CI, 1.9-2.3) (29). Between 2011-2015, overall antibiotic consumption in the European hospital sector (expressed as DDD per 1,000 inhabitants and per day) showed no significant trend (40). There was also no significant trend observed in the consumption of antibiotics for treatment of patients 
infected with serious multidrug-resistant (MDR) bacteria during this period at European level (1). In 2015, the European population-weighted mean consumption of antibiotics for systemic use in the hospital sector was 2,000 DDD/1000 PD, ranging from 1.0 in the Netherlands to 2.9 in Malta (40). However, no ECDC surveillance data specifically analyses the trend of antibiotic consumption in VAP patients.

Magill et al. (41) conducted a cross-sectional evaluation of antimicrobial use in US acute care hospitals, assessing it over a 2-day period in 10 American states between May and September 2011. Hospital antibiotic use prevalence was $49.9 \%$ (95\% CI, 49.0-50.9\%) with higher values in ICUs than in other locations $(57.7 \%, 95 \% \mathrm{CI}, 55.4-60.0 \%$ versus 48.6\%, 95\% CI, 47.6-49.6\%; $\mathrm{P}<0.001$ ). Baggs et al. (42) estimated trends in antibiotic use in more than 300 US hospitals from 2006 to 2012. Overall rates of antibiotic use did not change significantly during the study period. Antibiotic administration prevalence was $55.1 \%$ and the rate of antibiotic use for all study years was 755 days of therapy per 1,000 patient days (DOT/1,000 PD), rising from 732.5 DOT/1,000 PD in 2006 to 767.5 DOT/1,000 PD in 2012. Antibiotic consumption for all study years was $52 \%$ greater in ICUs compared to noncritical care locations $(1,092$ versus 720 DOT/1,000 PD, $\mathrm{P}<0.001)$. As its European counterparts, no CDC surveillance data specifically analyses the trend of antibiotic consumption in VAP patients.

\section{Discussion}

VAP has an undeniable clinical and epidemiological relevance and accounts for up to $50 \%$ of all antibiotics used in ICUs $(1,20,43)$. It would then be expected that VAP rates and antibiotic consumption evolved similarly, which surprisingly does not happen. The described limitations in the definition of VAP contribute to this disagreement but are not the only intervening factors.

Particular attention should be taken in interpreting studies with surprisingly low VAP rates. Such reports must be carefully analyzed in order to correctly access how artificial or manipulated the rates might be. Although VAP prevention bundles may contribute for encouraging reductions in VAP episodes, one should not lightly believe that a 'zero-VAP rate' is feasible (32). VAP pathophysiology and epidemiology limit its ability to prevent all episodes of VAP (32). Prevention bundles are essentially effective in reducing late onset VAP, that accounts for up to $60 \%$ of VAP (44), while early onset VAP, in turn, is mainly related to the presence of an endotracheal tube and the resulting violation of natural defense mechanisms (15). VAP risk factors such as each patient host flora, severity of underlying disease, microaspiration of oropharyngeal content (5) (particularly important in trauma patients when a low Glasgow Coma Score is present but also relevant whenever tracheal intubation was unscheduled and/or the patient was nonfasted), grade of functional immunosuppression when critically ill, previous surgery and antibiotic exposure (15) are all endogenous to patients, cannot be fully addressed by prevention bundles and may be unavoidable consequences of caring for critically ill patients $(17,18)$.

Besides variation in diagnostic and record practices, ICU characteristics and patient population, such as clinical severity and infection control guidelines may constitute surveillance biases and also affect the reported incidence of VAP (28).

The evaluation of antibiotic consumption is not uniform. The World Health Organization concept of DDD—the assumed maintenance dose per day in adults-can be used to standardize the comparisons of drug consumptions in various conditions (38) but its ratio per 1,000 patient-days is not used in all antibiotic surveillance reports; some authors presented antibiotic use by the ratio of patients treated with antibiotics and this difference in antibiotic quantification may contribute with confusing data. Using the DDD/1,000 PD ratio may itself constitute a bias because, although describing antibiotic pressure it does not describe accurately the number of patients treated and those not treated with antibiotic during each period (38): the exact same DDD/1,000 PD ratio may represent a small number of patients receiving a class of antibiotics for a long period or at a high dose as well as a large number of patients receiving small amounts of antibiotic or antibiotic over a short period $(1,38)$. Moreover, this method is influenced by the length of stay of patients and may overlook antibiotic de-escalation (45).

Knowledge and understanding of bacterial epidemiology and resistance trends for each ICU have great relevance. In general, late onset VAP is caused by multi-drug resistant and more difficult to treat bacteria than early onset VAP (15). Data from ECDC (28), NHSN (46), Canadian (33) and French (1) ICUs all report different bacteria isolation rates and resistance patterns which implies distinct initial empiric antibiotic and diverse global and group-specific antibiotic use. ECDC data from 2014 (47) and 2016 (28) reported that the most frequently isolated microorganisms in VAP were Pseudomonas aeruginosa followed by Staphylococcus aureus [with an average percentage of methicillin-resistant Staphylococcus aureus (MRSA) of 43.0\%], Klebsiella spp. and Escherichia coli. Fihman 
et al. (1) observed a significant increase in resistance rates to several classes of antimicrobial drugs in a retrospective 5-year trend analysis of VAP patients in a French tertiary-hospital ICU. Such resistance trend was more significant due to an increase in the isolation of AmpC-producing Enterobacteriaceae. Koulenti et al. (44) prospectively analyzed VAP patients from 27 ICUs in 9 European countries and isolated MDR organisms (MRSA, Pseudomonas aeruginosa, Acinetobacter baumannii and Stenotrophomonas maltophilia) in $53.7 \%$ of cases. Weiner et al. (46) described antimicrobial resistance patterns for VAPs reported to the NHSN from 2011 and 2012: the most isolated bacteria were Stapbylococcus aureus (percentage of MRSA 46.1\% in 2011 and 42.4\% in 2012) and Pseudomonas aeruginosa (percentage of MDR 20.8\% in 2011 and 19.9\% in 2012). Craven et al. (7) examined the incidence and microbiological data from VAT and VAP in medical and surgical ICU patients and MDR pathogens were isolated in $50 \%$ of VAP patients, namely MRSA that was present in more than $20 \%$ of VAP patients. The emergence over the last decade of MDR bacteria certainly contributed to an increase in antibiotic consumption, which itself increases the selective pressure favoring the most resistant bacteria $(1,46)$.

The new NHSN criteria provides a more objective and quantitative data based definition, that may theoretically, be advantageous. First, they can potentially reduce the methodological biases on IVAC rate calculation, providing trustworthy values for surveillance and benchmarking. Second, these new criteria provide clinicians with the capability of early recognizing and attending patients with mechanical ventilation-related problems that could be missed by VAP prevention or ancient surveillance protocols (17), thus taking the first step towards elucidating these events and creating opportunities to improve care. Third, the duty to include an antibiotic administration in IVAC definition, may supply reliable and comparable data on antibiotic consumption between ICUs (17). Quality indicators for infection control and antibiotic stewardship and/or surveillance programs will then have the possibility to become aware of IVAC-related antibiotic prescription, to identify outlier prescribers and to tailor antibiotic therapy, thereby reducing the risk of MDR bacteria emergence. All this may allow a more comprehensive assessment of the observed VAP and antibiotic consumption variability.

It is important though to realize that the new NHSN metrics are not free from potential biases. An IVAC can encompass both VAP and VAT clinical concepts since it does not use chest $\mathrm{X}$-ray as a diagnostic criterion and it is impossible to clinically distinguish VAP from VAT without it. It will then be possible to observe identical IVAC rates between studies but with differences between VAT and VAP numbers, which in addition to being discrepant may not be real: some patients who were diagnosed as having VAP may in fact have VAT and vice versa (5). Lachiewicz et al. (48) assessed the effect of the NHSN definition (possible VAP) on VAP rates in a burn ICU over different time periods (from July 2011 to December 2012 they used the old definition; from January 2013 to June 2014 and from July 2014 to December 2015 the new criteria were applied) and found fewer VAP cases (rates from 4.47 to 1.03 and $0.55 \mathrm{VAP} / 1,000$ ventilator days, respectively) with the new definition. No statistically significant differences were found between VAP groups regarding length of stay and mortality. However, when the ICU infection preventionists evaluated cases screened from July 2014 to December 2015 that did not meet possible VAP criteria, 18 VAP cases meeting the pre-2013 criteria were identified, resulting in an adjusted incidence of $4.96 \mathrm{VAP} / 1,000$ ventilator days. Younan et al. (10) compared the outcomes of patients meeting the classic VAP and/or the possible VAP criteria in a cohort of ICU trauma patients. A significant difference was found between classic VAP (30.9\%) and possible VAP (6.6\%) incidence and only $5.8 \%$ of patients $(n=1,116)$ met both definition criteria, with identical mortality. These results imply that the NHSN new surveillance definition may miss potentially clinically meaningful events that are important for driving infection prevention $(10,48)$, due to its low sensitivity and positive predictive value for identifying VAP (11).

\section{Conclusions}

The epidemiological relevance and clinical impact of VAP are unequivocal. It is therefore essential to ensure that objective and uniform definitions are used. VAP management must focus not only on early suspecting, diagnosing and properly treating but also on reliably recording epidemiological data (regarding both VAP incidence and antibiotic doses and duration of therapy) in order to assess and benchmark the quality of care for each type of ICU patients as accurately as possible. Microbiology epidemiological data-including prescription habits, the infections treated, infection control measures and epidemic phenomena between ICUs - are keystone for diagnosis and for tailoring antibiotic therapy. Appropriate antibiotics in adequate doses followed by de-escalation based on microbiological results and the clinical response of the patient constitute the best weapon against the potential 
for antibiotic overuse, the emergence of MDR bacteria, unnecessary adverse effects and potential toxicity. This must be the way to guarantee that there will be fewer discrepancies and more parallelism between VAP rate and antibiotic consumption in the near future.

\section{Acknowledgements}

None.

\section{Footnote}

Conflicts of Interest: The authors have no conflicts of interest to declare.

\section{References}

1. Fihman V, Messika J, Hajage D, et al. Five-year trends for ventilator-associated pneumonia: Correlation between microbiological findings and antimicrobial drug consumption. Int J Antimicrob Agents 2015;46:518-25.

2. Ferreira CR, Souza DF De, Cunha TM, et al. The effectiveness of a bundle in the prevention of ventilatorassociated pneumonia. Braz J Infect Dis 2016;20:267-71.

3. Skrupky LP, McConnell K, Dallas J, et al. A comparison of ventilator-associated pneumonia rates as identified according to the National Healthcare Safety Network and American College of Chest Physicians criteria. Crit Care Med 2012;40:281-4.

4. Gupta R, Malik A, Rizvi M, et al. Epidemiology of multidrug-resistant Gram-negative pathogens isolated from ventilator-associated pneumonia in ICU patients. J Glob Antimicrob Resist 2017;9:47-50.

5. Martin-Loeches I, Povoa P, Rodriguez A, et al. Incidence and prognosis of ventilator-associated tracheobronchitis (TAVeM): a multicentre, prospective, observational study. Lancet Respir Med 2015;3:859-68.

6. Centers for Disease Control and Prevention. VentilatorAssociated Event (VAE) Protocol [Internet] 2017. Available online: https://www.cdc.gov/nhsn/pdfs/pscmanual/10-vae_ final.pdf

7. Craven DE, Lei Y, Ruthazer R, et al. Incidence and outcomes of ventilator-associated tracheobronchitis and pneumonia. Am J Med 2013;126:542-9.

8. Craven DE, Hudcova J, Lei Y, et al. Pre-emptive antibiotic therapy to reduce ventilator-associated pneumonia: "thinking outside the box." Crit Care 2016;20:300.

9. Craven DE. Preventing Ventilator-Associated Pneumonia in
Adults - Sowing Seeds of Change. Chest 2006;130:251-60.

10. Younan D, Griffin R, Swain T, et al. Trauma patients meeting both Centers for Disease Control and Prevention's definitions for ventilator-associated pneumonia had worse outcomes than those meeting only one. J Surg Res 2017;216:123-8.

11. Klompas M, Berra L. Should Ventilator-Associated Events become a Quality Indicator for ICUs? Respir Care 2016;61:723-36.

12. Kobayashi H, Uchino S, Takinami M, et al. The Impact of Ventilator-Associated Events in Critically Ill Subjects With Prolonged Mechanical Ventilation. Respir Care 2017. [Epub ahead of print].

13. Klompas M. Interobserver variability in ventilatorassociated pneumonia surveillance. Am J Infect Control 2010;38:237-9.

14. Tejerina E, Esteban A, Fernández-Segoviano P, et al. Accuracy of Clinical Definitions of Ventilator-Associated Pneumonia: Comparison With Autopsy Findings. J Crit Care 2010;25:62-8.

15. Kalanuria AA, Zai W, Mirski M. Ventilator-associated pneumonia in the ICU. Crit Care 2014;18:208.

16. Centers for Disease Control and Prevention. Pneumonia (Ventilator-associated [VAP] and non-ventilatorassociated Pneumonia [PNEU]) Event [Internet] 2015. Available online: http://www.cdc.gov/nhsn/PDFs/ pscManual/6pscVAPcurrent.pdf

17. Klompas M. Complications of Mechanical Ventilation The CDC's New Surveillance Paradigm. N Engl J Med 2013;368:1472-5.

18. Mietto C, Pinciroli R, Pharmd NP, et al. Ventilator Associated Pneumonia : Evolving Definitions and Preventive Strategies. Respir Care 2013;58:990-1007.

19. Morris AC, Kefala K, Simpson AJ, et al. Evaluation of the effect of diagnostic methodology on the reported incidence of ventilator-associated pneumonia. Thorax 2009;64:516-22.

20. Klompas M, Khan Y, Kleinman K, et al. Multicenter evaluation of a novel surveillance paradigm for complications of mechanical ventilation. PLoS One 2011;6:1-7.

21. Bor C, Demirag K, Okcu O, et al. Ventilator-associated pneumonia in critically ill patients with intensive antibiotic usage. Pak J Med Sci 2015;31:1441-6.

22. Rosenthal VD, Al-Abdely HM, El-Kholy AA, et al. International Nosocomial Infection Control Consortium report, data summary of 50 countries for 20102015: Device-associatedmodule. Am J Infect Control 2016;44:1495-504. 
23. Rodríguez A, Póvoa P, Nseir S, et al. Incidence and diagnosis of ventilator-associated tracheobronchitis in the intensive care unit: an international online survey. Crit Care 2014;18:R32.

24. Dudeck MA, Weiner LM, Allen-Bridson K, et al. National Healthcare Safety Network (NHSN) report, data summary for 2012 , Device-associated module. Am J Infect Control 2013;41:1148-66.

25. VAP incidence rates by ICU type [Internet]. [cited 2017 Jun 22]. Available online: https://shea.confex.com/ data/abstract/shea/2010/Paper_1745_abstract_734_0. gif $\% 0 \mathrm{D} \% 0 \mathrm{~A}$

26. Dudeck MA, Horan TC, Peterson KD, et al. National Healthcare Safety Network (NHSN) Report, data summary for 2010, device-associated module. Am J Infect Control 2011;39:798-816.

27. Dudeck MA, Horan TC, Peterson KD. National Healthcare Safety Network (NHSN) Report, Data Summary for 2011, Device-associated Module. Am J Infect Control 2013;41:286-300.

28. European Centre for Disease Prevention and Control. Annual Epidemiological Report 2016 - Healthcareassociated infections acquired in intensive care units. Stockholm: ECDC; 2016. Available online: https://ecdc. europa.eu/en/publications-data/health

29. European Centre for Disease Prevention and Control. Point prevalence survey of healthcare- associated infections and antimicrobial use in European acute care hospitals. Stockholm: ECDC; 2013.

30. European Centre for Disease Prevention and Control. Surveillance of healthcare-associated infections in Europe, 2007. Stockholm: ECDC; 2012. Available online: ecdc. europa.eu/en/publications/Publications/120215_SUR_ HAI_2007.pdf

31. Klompas M. Eight initiatives that misleadingly lower ventilator-associated pneumonia rates. Am J Infect Control 2012;40:408-10.

32. Lisboa T, Rello J. Towards zero rate in healthcareassociated infections: one size shall not fit all .... Crit Care 2013;17:139.

33. Chin T, Kushner B, DerschMills D, et al. Antibiotic Utilization Patterns in Patients with Ventilator-Associated Pneumonia: A Canadian Context. Can J Infect Dis Med Microbiol 2016;2016:3702625.

34. Arthur LE, Kizor RS, Selim AG, et al. Antibiotics for ventilator-associated pneumonia. Cochrane Database Syst Rev 2016;10:CD004267.

35. Kalil AC, Metersky ML, Klompas M, et al. Management of Adults With Hospital-acquired and Ventilator-associated Pneumonia: 2016 Clinical Practice Guidelines by the Infectious Diseases Society of America and the American Thoracic Society. Clin Infect Dis 2016;63:e61-111.

36. Zilahi G, McMahon MA, Povoa P, et al. Duration of antibiotic therapy in the intensive care unit. J Thorac Dis 2016;8:3774-80.

37. Rosenthal VD, Rodrigues C, Álvarez-Moreno C, et al. Effectiveness of a multidimensional approach for prevention of ventilator-associated pneumonia in adult intensive care units from 14 developing countries of four continents: Findings of the International Nosocomial Infection Control Consortium. Crit Care Med 2012;40:3121-8.

38. Su SY, Chao C, Lai CC. The Association Between Antibiotic Consumption and the Rate of VentilatorAssociated Pneumonia in the ICU. Crit Care Med 2013;41:e53-4.

39. Hanberger H, Arman D, Gill H, et al. Surveillance of microbial resistance in European Intensive Care Units: a first report from the Care-ICU programme for improved infection control. Intensive Care Med 2009;35:91-100.

40. European Centre for Disease Prevention and Control. Summary of the latest data on antibiotic consumption in the European Union. European Surveillance of Antimicrobial Consumption Network surveillance data. Stockohlm: ECDC; 2016. Available online: http://ecdc. europa.eu/en/eaad/Documents/EARS-Net-summaryantibiotic-resistance.pdf

41. Magill SS, Edwards JR, Beldavs ZG, et al. Prevalence of Antimicrobial Use in US Acute Care Hospitals, MaySeptember 2011. JAMA 2014;312:1438-46.

42. Baggs J, Fridkin SK, Pollack LA, et al. Estimating National Trends in Inpatient Antibiotic Use Among US Hospitals From 2006 to 2012. JAMA Intern Med 2016;176:1639-48.

43. Chastre J, Fagon J. State of the Art: Ventilatorassociated Pneumonia Jean. Am J Respir Crit Care Med 2002;165:867-903.

44. Koulenti D, Lisboa T, Brun-Buisson C, et al. Spectrum of practice in the diagnosis of nosocomial pneumonia in patients requiring mechanical ventilation in European intensive care units. Crit Care Med 2009;37:2360-8.

45. Berrington A. Antimicrobial prescribing in hospitals: be careful what you measure. J Antimicrob Chemother 2010;65:163-8.

46. Weiner LM, Webb AK, Limbago B, et al. AntimicrobialResistant Pathogens Associated With HealthcareAssociated Infections: Summary of Data Reported to 
the National Healthcare Safety Network at the Centers for Disease Control and Prevention, 2011 - 2014. Infect Control Hosp Epidemiol 2016;37:1288-301.

47. European Centre for Disease Prevention and Control. Annual epidemiological report 2014. Antimicrobial resistance and healthcare-associated infections. Stockholm:
ECDC, 2015.

48. Lachiewicz AM, Weber DJ, van Duin D, et al. From VAP to VAE: Implications of the New CDC Definitions on a Burn Intensive Care Unit Population. Infect Control Hosp Epidemiol 2017;38:867-9.
Cite this article as: Nora D, Póvoa P. Antibiotic consumption and ventilator-associated pneumonia rates, some parallelism but some discrepancies. Ann Transl Med 2017;5(22):450. doi: 10.21037/atm.2017.09.16 